\title{
PFIM 4.0, an extended R program for design evaluation and optimization in nonlinear mixed-effect models
}

\author{
Cyrielle Dumont ${ }^{1,2}$, Giulia Lestini ${ }^{1}$, Hervé Le Nagard ${ }^{1}$, France Mentré ${ }^{1}$, Emmanuelle Comets ${ }^{1}$, \\ Thu Thuy Nguyen ${ }^{1, *}$, for the PFIM group ${ }^{1}$ \\ ${ }^{1}$ IAME, UMR 1137, INSERM and University Paris Diderot, Sorbonne Paris Cité, F-75018 Paris, France \\ ${ }^{2}$ University of Lille, EA 2694, Public Health: Epidemiology and Healthcare quality, ILIS, F-59000 Lille, France
}

\begin{abstract}
Background and objective: Nonlinear mixed-effect models (NLMEMs) are increasingly used for the analysis of longitudinal studies during drug development. When designing these studies, the expected Fisher information matrix (FIM) can be used instead of performing time-consuming clinical trial simulations. The function PFIM is the first tool for design evaluation and optimization that has been developed in R. In this article, we present an extended version, PFIM 4.0, which includes several new features.

Methods: Compared with version 3.0, PFIM 4.0 includes a more complete pharmacokinetic / pharmacodynamic library of models and accommodates models including additional random effects for inter-occasion variability as well as discrete covariates. A new input method has been added to specify user-defined models through an R function. Optimization can be performed assuming some fixed parameters or some fixed sampling times. New outputs have been added regarding the FIM such as eigenvalues, conditional numbers, and the option of saving the matrix obtained after evaluation or optimization. Previously obtained results, which are summarized in a FIM, can be taken into account in evaluation or optimization of one-group protocols. This feature enables the use of PFIM for adaptive designs. The Bayesian individual FIM has been implemented, taking into account a priori distribution of random effects. Designs for maximum a posteriori Bayesian estimation of individual parameters can now be evaluated or optimized and the predicted shrinkage is also reported. It is also possible to visualize the graphs of the model and the sensitivity functions without performing evaluation or optimization.

Results: The usefulness of these approaches and the simplicity of use of PFIM 4.0 are illustrated by two examples: i) an example of designing a population pharmacokinetic study accounting for previous results, which highlights the advantage of adaptive designs; ii) an example of Bayesian individual design optimization for a pharmacodynamic study, showing that the Bayesian individual FIM can be a useful tool in therapeutic drug monitoring, allowing efficient prediction of estimation precision and shrinkage for individual parameters.
\end{abstract}

Conclusion: PFIM 4.0 is a useful tool for design evaluation and optimization of longitudinal studies in pharmacometrics and is freely available at http://www.pfim.biostat.fr.

Keywords: Design, D-optimality, Fisher information matrix, Nonlinear mixed-effect model, PFIM

\footnotetext{
${ }^{*}$ Corresponding author at: IAME, UMR 1137, INSERM - University Paris Diderot, 16 Henri Huchard, 75018, Paris, France. Tel: +33 1572775 35. E-mail address: thu-thuy.nguyen@inserm.fr (T.T. Nguyen).
} 


\section{Introduction}

Nonlinear mixed-effect models (NLMEMs) are frequently used in model-based drug development to analyze longitudinal data [1]. They were initially used in pharmacokinetic (PK) or pharmacodynamic (PD) analyses: PK analysis deals with the time course of drug concentration, whereas PD refers to the relationship between the drug effect and doses or concentrations. The analysis through NLMEMs (i.e. the population approach) allows the estimation of mean parameters, their inter-individual/inter-occasion variability as well as covariate effects, and is appropriate for exploiting the richness of repeated measurements. Consequently, this approach is increasingly used in the biomedical field, not only for PKPD [2, 3] or joint PK analysis of parent drugs and their active metabolites [4, 5], but also for analyses of viral loads [6], of bacterial resistance to antibiotics [7], and of the dose-response relationship [8]. This approach has become the main statistical tool in pharmacometrics, the science of quantitative pharmacology [9]. To estimate parameters in NLMEMs, maximum likelihood estimation is commonly used, although the likelihood for these models has no analytical solution. Specific algorithms, implemented in several software packages, have therefore been proposed to perform this maximization $[10,1]$. Once population parameters are estimated, individual parameters can be obtained using maximum a posteriori (MAP) Bayesian estimation. This approach optimally incorporates all the information available from the whole population to increase the ability to estimate individual parameters and allows the use of sparse sampling, where few samples are collected from each subject.

Before the estimation step, the investigator of a study is confronted with the choice of the experimental design, which is crucial for efficient estimation of parameters in NLMEMs, especially when the studies are conducted in patients from whom few samples can be taken. A design in NLMEMs, also called a population design, is composed of the number of elementary designs and the specification of each elementary design and the associated number of subjects. In this setting, the term elementary design is used to describe a group of subjects with identical design characteristics. The choice of design consists in determining a balance between the number of subjects and the number of samples per subject, as well as the allocation of informative times and doses, according to experimental constraints. To evaluate and compare designs, the theory of optimum experimental design in classic nonlinear models has been introduced [11, 12, 13], based on the expected Fisher information matrix (FIM). The inverse of the FIM, according to the Cramer-Rao inequality, is the 
lower bound of the variance covariance matrix of any unbiased estimators of the parameters. From the square roots of the diagonal elements of the inverse of the FIM, the predicted standard errors (SE) for estimated parameters can be calculated. A widely used optimality criterion for design optimization is the D-criterion, which consists in maximizing the determinant of the FIM. While the individual FIM for standard nonlinear regression has an analytical expression in fixed effect models, there is no closed form of the population FIM in NLMEMs. That is why linear approximations of the model are common approaches in the design theory to evaluate the population FIM $[14,15,16]$. When linearizing the model around a guess value of the fixed effects, the variance of the observations is then independent of the fixed effects, which leads to a block-diagonal expression of the FIM [17]. It has been shown that this simpler expression of the FIM performed better than the full matrix expression given by other linearization approaches, providing results closer to those obtained by clinical trial simulation [18]. This expression of the FIM was extended to design crossover trials, including inter-occasion variability and discrete covariates fixed or changing between periods [19]. Beside the individual and population FIM, the expected Bayesian individual FIM was also developed to predict the estimation error of individual parameters obtained by MAP [20, 21]. In addition, the Bayesian FIM can also be used to predict the shrinkage [21], a metric quantifying the informativeness of the individual data and the reliability of individual parameter estimates [22]. The design approach based on these developments using the FIM is a good alternative to clinical trial simulation [23]. However, it requires a priori knowledge of the model and its parameters, which can usually be obtained from previous experiments and which leads to locally optimal designs. Alternatives to locally optimal designs are robust designs, relying on a priori distribution of parameters [24, 25], or adaptive designs, which use accumulating information in order to decide how to modify predefined aspects of the study during its implementation instead of leaving them fixed until the end [26, 27]. An adaptive design approach in NLMEMs that optimizes the design of each cohort while taking into account previous FIM obtained from previous cohorts has been proposed [28, 29].

Expressions of the individual FIM and population FIM based on FO are available in several software tools for designs in NLMEMs [18]. In particular, these expressions were implemented in PFIM, the first R function dedicated to design evaluation and optimization (www.pfim.biostat.fr). PFIM has been available since 2001 [30] for designs in single-response models. Version 3.0 in- 
cluding extensions of PFIM to multiple-response models was released in 2008 and described in [31]. Then version 3.2 was released in 2010, with a more complete PKPD library of models, had additional features, as described in [32], for including inter-occasion variability (IOV), discrete covariates with prediction of power for the comparison or equivalence Wald test [33, 19]. PFIM Interface, the graphical user interface (GUI) using R software, is also available and can be used to perform several features of the R script versions of PFIM. Optimization in PFIM is based on the D-optimality criterion described previously. Version 3.0 and later versions implement two optimization algorithms in PFIM: Simplex [34] and the Fedorov-Wynn algorithm [35, 36, 33].

Several features have been added to the new version 4.0 of PFIM. This new version includes a new input method to specify user-defined models through an $\mathrm{R}$ function. Design optimization can now be performed with fixed parameters or fixed sampling times. The FIM obtained after evaluation or optimization can be saved in a file. Evaluation and optimization can also be performed accounting for a previous FIM which summarizes previously obtained results, following the principle of adaptive designs. Additional features based on the Bayesian individual FIM have been implemented. Designs for MAP estimation of individual parameters can be evaluated or optimized and the predicted shrinkage is also reported. Finally, it is now possible to visualize the graphs of the model and the sensitivity functions without necessarily performing evaluation or optimization.

All the new features of PFIM 4.0 are described in this article. Section 2 presents the methodological developments for different new aspects of designs in NLMEMs. Then, the features implemented in PFIM 4.0 and the structure of the R program and its use are presented in Section 3, through a summary of model specifications as well as a description of the input/output files. Lastly, two illustrations of the use of PFIM 4.0 are provided in Section 4: i) an example of designing a population PK study taking into account previous results and ii) an example of Bayesian individual design optimization for a dose-response study.

\section{Statistical methods}

\subsection{Design}

The elementary design $\xi_{i}$ of individual $i(i=1, \ldots, N)$ is defined by the number $n_{i}$ of samples and their allocation in time $\left(t_{i 1} \ldots, t_{i n_{i}}\right)$. In the case of $K$ responses, $\xi_{i}$ is composed of $K$ sub-designs 
such that $\xi_{i}=\left(\xi_{i 1}, \xi_{i 2}, \ldots, \xi_{i K}\right)$. The sub-design $\xi_{i k}$ is then defined by $\left(t_{i k 1}, t_{i k 2}, \ldots, t_{i k n_{i k}}\right)$, with $n_{i k}$ sampling times for the observations of the $k^{\text {th }}$ response, so that $n_{i}=\sum_{k=1}^{K} n_{i k}$.

In the case of designs with $H$ occasions, $\xi_{i}$ is composed of $H$ sub-designs such that $\xi_{i}=$ $\left(\xi_{i 1}, \xi_{i 2}, \ldots, \xi_{i H}\right)$. The design $\xi_{i h}$ at each occasion $h(h=1, \ldots, H)$ for $K$ responses is composed of $\left(\xi_{i h 1}, \xi_{i h 2}, \ldots, \xi_{i h K}\right)$, with $\xi_{i h k}=\left(t_{i h k 1}, t_{i h k 2}, \ldots, t_{i h k n_{i h k}}\right)$. The number of sampling times at the $h^{\text {th }}$ occasion is $n_{i h}=\sum_{k=1}^{K} n_{i h k}$, so that $n_{i}=\sum_{h=1}^{H} \sum_{k=1}^{K} n_{i h k}$.

For $N$ individuals, the population design is composed of the $N$ elementary designs such as $\Xi=\left\{\xi_{1}, \ldots, \xi_{N}\right\}$. Usually, population designs are composed of a limited number $Q$ of groups of individuals with identical design $\xi_{q}$ within each group, performed in a number $N_{q}$ of individuals. The population design can thus be written as $\Xi=\left\{\left[\xi_{1}, N_{1}\right] ;\left[\xi_{2}, N_{2}\right] ; \ldots ;\left[\xi_{Q}, N_{Q}\right]\right\}$. In the case of identical elementary designs in all individuals, the one-group population design is defined by $\Xi=$ $\{\xi, N\}$.

Individual design (for standard nonlinear regression) and individual Bayesian design (for Bayesian estimation of individual parameters) are defined only by an elementary design $\xi$ to be performed in one individual.

\subsection{Nonlinear mixed-effects models}

\subsubsection{Basic single and multiple response models}

An NLMEM, or a population model, with no covariate and for one response, is defined as follows. The vector of observations $Y_{i}$ for the individual $i(i=1, \ldots, N)$ is defined as:

$$
Y_{i}=f\left(\theta_{i}, \xi_{i}\right)+\varepsilon_{i}=f\left(g\left(\mu, b_{i}\right), \xi_{i}\right)+\varepsilon_{i},
$$

where the function $f$ defines the nonlinear structural model for one response, $\theta_{i}$ is the vector of the $p$-individual parameters for individual $i, \xi_{i}$ is the elementary design of individual $i$ and $\varepsilon_{i}$ is the vector of residual error. The vector of individual parameters $\theta_{i}$ depends on $\mu$, the $p$-vector of the fixed effect parameters and on $b_{i}$, the $p$-vector of the random effects for individual $i$ through a function $g$, that is $\theta_{i}=g\left(\mu, b_{i}\right)$. If $\theta_{i}$ follows a normal distribution then $g$ is an additive function, so that $\theta_{i}=\mu+b_{i}$. If $\theta_{i}$ follows a lognormal distribution then $g$ is an exponential function, so that $\theta_{i}=\mu \exp \left(b_{i}\right)$. 
It is assumed that $b_{i} \sim N(0, \Omega)$ with $\Omega$ defined as a $p \times p$ diagonal variance-covariance matrix, for which each diagonal element $\omega_{j, j=1, \ldots, p}$, represents the inter-individual variability of the $j^{t h}$ component of the vector $b_{i}$. It is also supposed that $\varepsilon_{i}$ follows a normal distribution of mean 0 and $n_{i} \times n_{i}$-diagonal variance-covariance matrix $\Sigma_{i}$. Conditional on the values of the individual parameters $\theta_{i}$, the matrix $\Sigma_{i}$ is defined as $\Sigma_{i}\left(\theta_{i}, \sigma_{\text {inter }}, \sigma_{\text {slope }}, \xi_{i}\right)=\operatorname{diag}\left(\sigma_{\text {inter }}+\sigma_{\text {slope }} f\left(\theta_{i}, \xi_{i}\right)\right)^{2}$. The terms $\sigma_{\text {inter }}$ and $\sigma_{\text {slope }}$ are the additive and proportional parts of the error model, respectively. Of note $\Sigma_{i}=\sigma_{i n t e r}^{2} I_{n_{i}}$ for a additive error variance model, $\Sigma_{i}=\operatorname{diag}\left(\sigma_{\text {slope }} f\left(\theta_{i}, \xi_{i}\right)\right)^{2}$ for a proportional error variance model and $\Sigma_{i}=\operatorname{diag}\left(\sigma_{\text {inter }}+\sigma_{\text {slope }} f\left(\theta_{i}, \xi_{i}\right)\right)^{2}$ for a combined error variance model. Conditional upon the value of $b_{i}$, it is assumed that the $\varepsilon_{i}$ errors are independently distributed.

In the case of $K$ multiple responses, the vector of observations $Y_{i}$ can then be composed of $K$ vectors for the different responses:

$$
Y_{i}=\left[Y_{i 1}^{T}, Y_{i 2}^{T}, \ldots, Y_{i K}^{T}\right]^{T}
$$

where $Y_{i k}, k=1, \ldots, K$, is the vector of $n_{i k}$ observations for the $k^{t h}$ response. Each of these responses is associated with a known function $f_{k}$, which can be grouped in a vector of a multiple response model $F$, such as

$$
F\left(\theta_{i}, \xi_{i}\right)=\left[f_{1}\left(\theta_{i}, \xi_{i 1}\right)^{T}, f_{2}\left(\theta_{i}, \xi_{i 2}\right)^{T}, \ldots, f_{K}\left(\theta_{i}, \xi_{i K}\right)^{T}\right]^{T}
$$

Each response can have its error model and $\varepsilon_{i}$ is then the vector composed of the $K$ vectors of residual errors $\varepsilon_{i k}, k=1, \ldots, K$, associated with the $K$ responses. It is also supposed that $\varepsilon_{i k} \sim N\left(0, \Sigma_{i k}\right)$ with $\Sigma_{i k}$ a $n_{i k} \times n_{i k}$-diagonal matrix such that: conditional on the values of $\theta_{i}$, $\Sigma_{i k}\left(\theta_{i}, \sigma_{\text {inter }_{k}}, \sigma_{\text {slope }_{k}}, \xi_{i k}\right)=\operatorname{diag}\left(\sigma_{\text {inter }_{k}}+\sigma_{\text {slope }_{k}} f_{k}\left(\theta_{i}, \xi_{i k}\right)\right)^{2}$ where $\sigma_{\text {inter }_{k}}$ and $\sigma_{\text {slope }_{k}}$ are two parameters of the model for the variance of the residual error of the $k^{\text {th }}$ response. The variance of $\varepsilon_{i}$ over the $K$ responses is then noted $\Sigma_{i}\left(\theta_{i}, \sigma_{\text {inter }}, \sigma_{\text {slope }}, \xi_{i}\right)$, where $\Sigma_{i}$ is a $n_{i} \times n_{i}$-diagonal matrix composed of each diagonal element of $\Sigma_{i k}$ with $k=1, \ldots, K$ and $\sigma_{\text {inter }}$ and $\sigma_{\text {slope }}$ are two vectors of the $K$ components $\sigma_{\text {inter }_{k}}$ and $\sigma_{\text {slope }_{k}}, k=1, \ldots, K$, respectively. 


\subsubsection{Models accounting for inter-occasion variability}

In mixed models, several levels of variability can be taken into account. In particular, in the case of trials including several occasions, there are additional random effects for inter-occasion variability that can be estimated separately from inter-individual variability. For instance, the individual parameters $\theta_{i h}$ of an individual $i$ at occasion $h$ are linked to the fixed effect $\mu$, the inter-individual variability random effects $b_{i}$ and the inter-occasion variability random effects $\kappa_{i h}$ through the function $g$ defined previously, that is $\theta_{i h}=g\left(\mu, b_{i}+\kappa_{i h}\right) . b_{i}$ and $\kappa_{i h}$ are supposedly independent. It is assumed that $b_{i} \sim N(0, \Omega)$ and $\kappa_{i h} \sim N(0, \Gamma)$ with $\Omega$ and $\Gamma$ defined as diagonal matrices of size $p \times p$. Each element $\omega_{j}$ of $\Omega$ and $\gamma_{j}$ of $\Gamma$ represents the inter-individual variability of the $j^{\text {th }}$ component of $b_{i}$ and the inter-occasion variability of the $j^{t h}$ component of $\kappa_{i h}$, respectively.

\subsubsection{Models accounting for discrete covariates}

Part of the inter-individual variability or inter-occasion variability could be explained by the influence of covariates which can be continuous or discrete and can also change between occasions. Only discrete covariates will be addressed in this work.

Let $C$ denote the set of discrete covariates changing or not between occasions, and $D_{c}$ is the set of all possible categories $d$ of the covariate $c$ in $C$. It is assumed either that covariates are additive for parameters if the random effect model is additive, or that covariates are additive for log parameters if the random effect model is exponential. For instance, in the case of an exponential model, the individual parameter vector $\theta_{i h}$ for an individual $i$ at occasion $h$ can be expressed as a function of the covariate $c_{i h}$ as follows:

$$
\theta_{i h}=\mu \times \exp \left(\sum_{c \in C} \sum_{d \in D_{c}} 1_{c_{i h}=d} \beta_{c_{d}}\right) \times \exp \left(b_{i}+\kappa_{i h}\right) .
$$

The covariate $c_{i h}$ for individual $i$ at occasion $h$ can take any value $d$ in the set $D_{c} .1_{c_{i h}=d}$ is the indicator function of the category $d$ in the set $D_{c}$. $\beta_{c_{d}}$ is the $p$-vector of effects for category $d$ of covariate $c$ ( $\beta_{c_{d}}=0$ for the category of reference) for the $p$ parameters of the structural model. We denote $\rho$ the vector of all fixed effects composed of $\mu$ and $\beta_{c_{d}}$ and $u$ the function characterizing their relationship with the vector of all covariates $C_{i h}$ such as $\theta_{i h}=g\left(u\left(\rho, C_{i h}\right), b_{i}+\kappa_{i h}\right)$. 


\subsection{Fisher information matrix}

Three types of FIM are proposed in this paper to quantify the informativeness of the designs, according to different analysis purposes: the population FIM, $M_{P F}(\Psi, \Xi)$, for population analysis, the individual FIM, $M_{I F}\left(\theta_{i}, \xi_{i}\right)$, for individual regression and the individual Bayesian FIM, $M_{B F}\left(\xi_{i}\right)$, for Bayesian estimation of individual parameters in NLMEMs. The population Bayesian FIM for population analysis is not addressed in the present work.

\subsubsection{Population Fisher information matrix}

\section{Expression of $M_{P F}$}

We consider the model $Y_{i}=F\left(\theta_{i}, \xi\right)+\varepsilon_{i} . \theta_{i}=\left(\theta_{i 1}^{T}, \ldots, \theta_{i H}^{T}\right)^{T}$ is the individual parameter vector for $H$ occasions. $\theta_{i} \sim N\left(u_{i}(\rho), \Omega^{*}\right)$ in the case of additive random effects and $\log \theta_{i} \sim N\left(\log u_{i}(\rho), \Omega^{*}\right)$ in the case of exponential random effects, with $u_{i}(\rho)=\left(u\left(\rho, C_{i 1}\right)^{T}, \ldots, u\left(\rho, C_{i H}\right)^{T}\right)^{T}$ and $\Omega^{*}$ is the $H \times H$-blocks matrix with $(\Omega+\Gamma)$ on the diagonal and $\Omega$ elsewhere. We denote $\lambda$ the vector of the variance terms composed of the inter-individual variability and inter-occasion variability as well as residual variability. Let $\Psi$ be the population parameter vector defined by $\Psi^{T}=\left(\rho^{T}, \lambda^{T}\right)$. Assuming independence across $N$ individuals, the population $\operatorname{FIM,~} M_{P F}(\Psi, \Xi)$, for a population design $\Xi$, is defined by the sum of the $N$ elementary matrices $M_{P F}\left(\Psi, \xi_{i}\right)$ computed for each individual $i$,

$$
M_{P F}(\Psi, \Xi)=\sum_{i=1}^{N} M_{P F}\left(\Psi, \xi_{i}\right)
$$

For one subject, the elementary FIM, $M_{P F}\left(\Psi, \xi_{i}\right)$, for the elementary design $\xi_{i}$, is defined as

$$
M_{P F}\left(\Psi, \xi_{i}\right)=E_{Y_{i}}\left(\frac{\partial \log L\left(\Psi, Y_{i}\right)}{\partial \Psi} \frac{\partial \log L\left(\Psi, Y_{i}\right)^{T}}{\partial \Psi}\right)
$$

where $L\left(\Psi, Y_{i}\right)$ is the likelihood of the observations $Y_{i}$ given the population parameters $\Psi$. Under some general regularity conditions, equation (6) is equivalent to $M_{P F}\left(\Psi, \xi_{i}\right)=E_{Y_{i}}\left(-\frac{\partial^{2} \log L\left(\Psi, Y_{i}\right)}{\partial \Psi^{2}}\right)$. Linear approximations of the model are common approaches in the design theory to circumvent the problem of the missing closed form of the likelihood [14]. By linearizing the response function $F$ around a guess value of the fixed effects $u_{i}\left(\rho_{0}\right)$ in the case of additive random effects, as in [17], 
we obtain (subscript $i$ omitted in the followings):

$$
\begin{aligned}
Y & \simeq F\left(u\left(\rho_{0}\right), \xi\right)+\left.\frac{\partial F(\theta), \xi)}{\partial \theta^{T}}\right|_{\theta=u\left(\rho_{0}\right)}\left(\theta-u\left(\rho_{0}\right)\right)+\varepsilon \\
& \simeq F\left(u\left(\rho_{0}\right), \xi\right)+\left.\frac{\partial F(\theta, \xi)}{\partial \theta^{T}}\right|_{\theta=u\left(\rho_{0}\right)}(\theta-u(\rho))+\left.\frac{\partial F(\theta, \xi)}{\partial \theta^{T}}\right|_{\theta=u\left(\rho_{0}\right)}\left(u(\rho)-u\left(\rho_{0}\right)\right)+\varepsilon
\end{aligned}
$$

As a consequence, it is assumed $Y \sim N(E, V)$ with :

$$
\begin{aligned}
E(Y) \cong E= & F\left(u\left(\rho_{0}\right), \xi\right)+\left.\frac{\partial F(\theta, \xi)}{\partial \theta^{T}}\right|_{\theta=u\left(\rho_{0}\right)}\left(u(\rho)-u\left(\rho_{0}\right)\right) \\
\operatorname{Var}(Y) \cong V= & \left(\left.\frac{\partial F(\theta), \xi)}{\partial \theta^{T}}\right|_{\theta=u\left(\rho_{0}\right)}\right) \Omega^{*}\left(\left.\frac{\partial F(\theta), \xi)}{\partial \theta^{T}}\right|_{\theta=u\left(\rho_{0}\right)}\right)^{T} \\
& +\Sigma\left(u\left(\rho_{0}\right), \sigma_{\text {inter }}, \sigma_{\text {slope }}, \xi\right)
\end{aligned}
$$

By deriving the $\log$-likelihood $\log L(\Psi, Y) \simeq-\frac{1}{2} n \log (2 \pi)-\frac{1}{2} \log |V|-\frac{1}{2}(Y-E)^{T} V^{-1}(Y-E)$, the elementary FIM is a block matrix defined as

$$
M_{P F}\left(\Psi, \xi_{i}\right)=\frac{1}{2}\left(\begin{array}{ll}
A & C \\
C^{T} & B
\end{array}\right)
$$

where the blocks $A, B$ and $C$ have following expressions[37]:

$$
A_{m l} \cong 2 \frac{\partial E^{T}}{\partial \rho_{m}} V^{-1} \frac{\partial E}{\partial \rho_{l}}+\operatorname{tr}\left(\frac{\partial V}{\partial \rho_{m}} V^{-1} \frac{\partial V}{\partial \rho_{l}} V^{-1}\right)
$$

with $m$ and $l=1, \ldots, \operatorname{dim}(\rho)$

$$
B_{m l} \cong \operatorname{tr}\left(\frac{\partial V}{\partial \lambda_{m}} V^{-1} \frac{\partial V}{\partial \lambda_{l}} V^{-1}\right)
$$

with $m$ and $l=1, \ldots, \operatorname{dim}(\lambda)$

$$
C_{m l} \cong \operatorname{tr}\left(\frac{\partial V}{\partial \rho_{m}} V^{-1} \frac{\partial V}{\partial \lambda_{l}} V^{-1}\right)
$$

with $m=1, \ldots, \operatorname{dim}(\rho)$ and $l=1, \ldots, \operatorname{dim}(\lambda)$.

Since $\left.\frac{\partial F(\theta, \xi)}{\partial \theta^{T}}\right|_{\theta=u\left(\rho_{0}\right)}$ is independent of $\rho, \frac{\partial V}{\partial \rho_{m}}=0$, the elementary FIM is then a block diagonal matrix with $A_{m l} \cong 2 \frac{\partial E^{T}}{\partial \rho_{m}} V^{-1} \frac{\partial E}{\partial \rho_{l}}$ in equation (10) and $C_{m l}=0$ in equation (12). Similar 
calculations can be done to evaluate the FIM in the case of exponential random effects by writing the model as $Y_{i}=F\left(\exp \left(\log \theta_{i}\right), \xi\right)+\varepsilon_{i}=G\left(\log \theta_{i}, \xi\right)+\varepsilon_{i}$ and by linearizing the response function $G$ around a guess value $\log u\left(\rho_{0}\right)$ for $\log \theta_{i}$. The FIM is then evaluated assuming $\rho_{0}=\rho$, the fixed effect values for which the design is evaluated. This block diagonal expression of the FIM is the one computed by default in PFIM. Other linearization approach to evaluate the FIM is also available, giving a full matrix expression of the FIM [18]. However the full matrix is not recommended, providing predicted standard errors which are less accurate than those obtained by the block diagonal matrix when comparing with clinical trial simulation results $[17,18]$.

Recently, new alternative methods have been developed (not available in PFIM) to compute the FIM for discrete data without linearization, using Adaptive Gaussian Quadrature (AGQ) [38] or Markov Chain Hamiltonian Monte Carlo (HMC) [39] to integrate the derivatives of the loglikelihood over the random effects, and Monte Carlo (MC) integration to evaluate its expectation with respect to the observations. The HMC-based approach is available in the R package MIXFIM [39].

\section{Computation of power and number of subjects needed using $M_{P F}$}

In this paragraph, we consider the Wald tests of comparison or equivalence on a discrete covariate effect, fixed or changing between occasions. These tests are performed on the estimator of the effect size $\beta_{c_{d}, p}$ of a category $d$ of each covariate $c$ for parameter $p$. Given a design and values of population parameters including a value for $\beta_{c_{d}, p}$, from $M_{P F}(\Psi, \Xi)$, we can derive the SE of $\beta_{c_{d}, p}$. Then, we can predict the power of the tests and also compute the number of subjects needed to achieve a given power $[33,19]$. For simplicity, we omit index $\left(c_{d}, p\right)$ for $\beta$ in this section.

The null hypothesis for the Wald test of comparison is $H_{0}:\{\beta=0\}$ while the alternative hypothesis is $H_{1}:\{\beta \neq 0\}$. Denoting $\widehat{\beta}$ as the estimator of $\beta$, then the statistic of the Wald test under the null hypothesis $H_{0}$ is $W=\widehat{\beta} / \operatorname{SE}(\widehat{\beta})$. With a type I error $\alpha, H_{0}$ is rejected if $|W|>z_{1-\alpha / 2}$, where $z_{1-\alpha / 2}$ is the $(1-\alpha / 2)$ quantile of the standard normal distribution.

We compute the power of the Wald test of comparison under $H_{1}$, when $\beta=\beta_{1} \neq 0$. Under $H_{1}$, $W$ is asymptotically distributed with a normal distribution centered at $\beta_{1} / \mathrm{SE}\left(\beta_{1}\right)$. Therefore, the power of the comparison Wald test $P_{C}$ is

$$
P_{C}=1-\Phi\left(z_{1-\alpha / 2}-\frac{\beta_{1}}{\operatorname{SE}\left(\beta_{1}\right)}\right)+\Phi\left(-z_{1-\alpha / 2}-\frac{\beta_{1}}{\operatorname{SE}\left(\beta_{1}\right)}\right)
$$


where $\Phi$ is the cumulative distribution function of the standard normal distribution.

In order to derive the number of subjects needed to achieve a power $P_{C}$ of the Wald test of comparison to detect an effect of a category versus the reference category for a covariate, we first compute the $\mathrm{SE}$ in $\beta_{1}$ needed to obtain this power, called $\operatorname{SEN}\left(P_{C}\right)$, using the following relation

$$
\operatorname{SEN}\left(P_{\mathrm{C}}\right)=\frac{\beta_{1}}{z_{1-\alpha / 2}-\Phi^{-1}\left(1-P_{\mathrm{C}}\right)}
$$

We then compute the the number of subjects needed to obtain a power of $P_{C}$, called NSN $\left(P_{\mathrm{C}}\right)$ using

$$
\operatorname{NSN}\left(P_{\mathrm{C}}\right)=N \times\left(\frac{\operatorname{SE}\left(\beta_{1}\right)}{\operatorname{SEN}\left(P_{\mathrm{C}}\right)}\right)^{2} .
$$

The null hypothesis for the Wald test of equivalence is $H_{0}:\{\beta \leq-\delta$ or $\beta \geq+\delta\}$, while the alternative hypothesis is $H_{1}:\{-\delta<\beta<+\delta\}$, where $\delta$ is the equivalence limit. In the guidelines for equivalence assessment $[40,41]$, it is recommended that $\delta=0.2 . H_{0}$ is composed of two unilateral hypotheses $H_{0,-\delta}:\{\beta \leq-\delta\}$ and $H_{0,+\delta}:\{\beta \geq+\delta\}$. Equivalence between two groups for the covariate effect $\beta$ can be concluded if and only if the two hypotheses $H_{0,-\delta}$ and $H_{0,+\delta}$ are rejected. The equivalence Wald test using NLMEMs has already been developed from [42], based on Schuirmann's two one-sided tests (TOST) [43]. Denoting the estimator of $\beta$ by $\widehat{\beta}$, the following formulas define $W_{-\delta}$, the statistic of the unilateral test under the null hypothesis $H_{0,-\delta}$, and $W_{+\delta}$, the statistic of the unilateral test under the null hypothesis $H_{0,+\delta}: W_{-\delta}=(\widehat{\beta}+\delta) / \operatorname{SE}(\widehat{\beta})$ and $W_{+\delta}=(\widehat{\beta}-\delta) / \operatorname{SE}(\widehat{\beta})$. With a type I error $\alpha, H_{0}$ is rejected if $W_{-\delta} \geq z_{1-\alpha}$ and $W_{+\delta} \leq-z_{1-\alpha}$, where $z_{1-\alpha}$ is the $(1-\alpha)$ quantile of the standard normal distribution.

We compute the power of the Wald test of equivalence under $H_{1}$, when $\beta=\beta_{1} \in[-\delta,+\delta]$. Usually we choose $\beta_{1}=0$. From a given design and values of population parameters, $S E\left(\beta_{1}\right)$ can be predicted using $M_{P F}$. The power of the equivalence Wald test $P_{E}$ is then

$$
\begin{array}{ll}
P_{\mathrm{E}}=1-\Phi\left(z_{1-\alpha}-\frac{\beta_{1}+\delta}{\mathrm{SE}\left(\beta_{1}\right)}\right) & \text { if } \beta_{1} \in[-\delta, 0], \\
P_{\mathrm{E}}=\Phi\left(-z_{1-\alpha}-\frac{\beta_{1}-\delta}{\mathrm{SE}\left(\beta_{1}\right)}\right) & \text { if } \beta_{1} \in[0,+\delta]
\end{array}
$$

where $\Phi$ is defined as previously. As expressed in (16) and (17), the power of the equivalence test 
depends on the sign of $\beta_{1}$. When $\beta_{1}=0$, we can use any of the two equations to obtain the power because in that case:

$$
P_{\mathrm{E}}=1-\Phi\left(z_{1-\alpha}-\frac{\delta}{\mathrm{SE}\left(\beta_{1}\right)}\right)=\Phi\left(-z_{1-\alpha}-\frac{(-\delta)}{\mathrm{SE}\left(\beta_{1}\right)}\right)
$$

In order to derive the number of subjects needed to achieve the power $P_{E}$ of showing the equivalence between a category versus the reference category for a covariate, we first compute the SE in $\beta_{1}$ needed to obtain the power of $P_{E}$, called $\operatorname{SEN}\left(P_{\mathrm{E}}\right)$, using

$$
\begin{array}{rlr}
\operatorname{SEN}\left(P_{\mathrm{E}}\right) & =\frac{-\beta_{1}-\delta}{-z_{1-\alpha}+\Phi^{-1}\left(1-P_{\mathrm{E}}\right)} & \text { if } \beta_{1} \in[-\delta, 0], \\
\operatorname{SEN}\left(P_{\text {equi }}\right) & =\frac{-\beta_{1}+\delta}{z_{1-\alpha}+\Phi^{-1}\left(P_{\mathrm{E}}\right)} & \text { if } \beta_{1} \in[0,+\delta],
\end{array}
$$

We then compute the number of subjects needed to obtain the power of $P_{E}$, called NSN $\left(P_{\mathrm{E}}\right)$ using (15) with $\operatorname{SEN}\left(P_{\mathrm{E}}\right)$ instead of $\operatorname{SEN}\left(P_{\mathrm{C}}\right)$. If $\beta_{1}<0$, we substitute (19) for (15); if $\beta_{1}>0$, we substitute (20) for (15); if $\beta_{1}=0$, we can substitute either of (19) or (20) for (15) because in that case:

$$
\operatorname{SEN}\left(P_{\mathrm{E}}\right)=\frac{-\delta}{-z_{1-\alpha}+\Phi^{-1}\left(1-P_{\mathrm{E}}\right)}=\frac{\delta}{z_{1-\alpha}+\Phi^{-1}\left(P_{\mathrm{E}}\right)} .
$$

\section{$M_{P F}$ accounting for previous results}

When evaluating or optimizing designs for maximum likelihood estimation of parameters $\Psi$, one may want to take into account results already obtained after fitting the data from a previous study or a previous cohort, which is usually done in adaptive designs. Following the approach proposed by [28] and [29], the previous results summarized in a predicted or an observed FIM, denoted $M_{P F_{\text {prev }}}(\Psi)$ could be combined with the current $M_{P F}(\Psi, \Xi)$ as follows:

$$
M_{P F}(\Psi, \Xi)+M_{P F_{p r e v}}(\Psi)
$$

In this formula, the previous FIM $M_{P F_{\text {prev }}}(\Psi)$ should have the same dimension as the current FIM $M_{P F}(\Psi, \Xi)$. 


\subsubsection{Individual and Bayesian Fisher information matrix}

In standard nonlinear regression models, the predicted individual FIM for individual parameter $\theta$ (index $i$ omitted for simplicity) is defined by

$$
M_{I F}(\theta, \xi)=E_{Y}\left(\frac{\partial \log p(Y \mid \theta)}{\partial \theta} \frac{\partial \log p(Y \mid \theta)^{T}}{\partial \theta}\right)
$$

where $p(Y \mid \theta)$ is the probability density of the observations $Y$ given the parameters $\theta$.

In NLMEMs, once population parameters are estimated, individual parameters can be derived using MAP, as the mode of the posteriori distribution. To find informative designs for MAP estimation, Bayesian experimental design approaches and Bayesian optimality criteria can be used $[44,45]$. For MAP estimation, $\mu$ is known, therefore estimating $\theta$ is similar to estimating the random effects $b$. More precisely, the MAP estimate of $b$ is given by $\hat{b}=\operatorname{argmax}(p(b \mid y))=$ $\operatorname{argmax}\left(\frac{p(y \mid b) p(b)}{p(y)}\right)=\operatorname{argmax}(\log (p(y \mid b))+\log (p(b)))$, where $p(y \mid b)$ is the probability density of the observations given the random effects and $p(b)$ the probability density of the random effects, which follows the normal distribution $N(0, \Omega)$. Thus the Bayesian individual FIM [20, 21] is expressed as

$$
\begin{aligned}
M_{B F}(\xi) & =E_{Y}\left(\frac{\partial \log p(b \mid Y)}{\partial b} \frac{\partial \log p(b \mid Y)^{T}}{\partial b}\right) \\
& =E_{b}\left(E_{Y \mid b}\left(\frac{\partial \log p(Y \mid b)}{\partial b} \frac{\partial \log p(Y \mid b)^{T}}{\partial b}\right)\right)+E_{b}\left(\frac{\partial \log p(b)}{\partial b} \frac{\partial \log p(b)^{T}}{\partial b}\right) .
\end{aligned}
$$

We recognized in the first term of expression (25) the expectation of the predicted individual FIM over the distribution of the random effects. As $b$ follows a Gaussian distribution, the second term is the inverse of $\Omega^{-1}$. Therefore we can write

$$
M_{B F}(\xi)=E_{b}\left(M_{I F}(g(\mu, b), \xi)\right)+\Omega^{-1}
$$

The expectation $E_{b}\left(M_{I F}(g(\mu, b), \xi)\right)$ can be evaluated by first-order linearization of the model around the expectation of random effects (i.e., 0$)$ as $M_{I F}(g(\mu, 0), \xi)$. The shrinkage (Sh), quantified the ratio of the estimation variance predicted by $M_{B F}^{-1}(\xi)$ and the variance of the random effects, can therefore be calculated as the diagonal elements of the matrix $I-W(\xi)=M_{B F}(\xi)^{-1} \Omega^{-1}[21]$. 


\section{Implementation and use of PFIM 4.0 in $R$}

The methods described previously have been implemented in R [R Development Core Team, R: A Language and Environment for Statistical Computing, R Foundation for Statistical Computing, Vienna, Austria, 2004] (http://cran.r-project.org/), in version 4.0 of the function PFIM available at www.pfim.biostat.fr.

\subsection{Features of PFIM 4.0}

Compared with version 3.0 described in [31], PFIM 4.0 includes a more complete PKPD library of models as well as a new way to specify user-defined model through $\mathrm{R}$ functions (see details in Section 3.3 below). Regarding statistical models, PFIM 4.0 accommodates models including additional random effects for inter-occasion variability as well as discrete covariates. Users can specify either additive or exponential random effects. PFIM 4.0 only supports designs with the same sampling times in each occasion, which is the design usually used in bioequivalence crossover trials. The number of occasions, of covariates, of parameters associated with each covariate as well as the number of categories for each covariate that can be included in the models is not limited. Knowledge of distributions of covariates is, however, required for FIM computation and these distributions are supposedly independent. It is possible to compute the power of the Wald tests of comparison or of equivalence for a discrete covariate effect that can be fixed or changing between different periods of a crossover trial. The Wald test is performed on the effect size of each category for each covariate; the global Wald test on the vector including all effect coefficients of a covariate is not implemented in the present version.

Regarding the expression of the FIM, based on publications showing the better performance of the block diagonal expression compared with the full one with first-order linearization of the model $[17,18]$, by default the block diagonal matrix is used in PFIM 4.0. Population, individual and Bayesian individual designs for single and multiple-response models can be evaluated based on the computation of the population, individual and Bayesian individual FIM, respectively. The expected $\mathrm{SE}$ and relative standard error (RSE) for the population or individual parameters corresponding to each type of design are calculated from the square roots of the diagonal elements of the inverse of FIM. The user can choose to fix one or several model parameters in the computation of the FIM.

Two algorithms have been available in PFIM since version 3.0 [31] for design optimization: the 
Simplex and the Fedorov-Wynn algorithm. The D-optimality criterion was used i.e the determinant of the FIM normalized by the number of parameters $P$ to be estimated in the model $\left(\operatorname{det}(F I M)^{1 / P}\right)$. The optimal design is then the one maximizing the determinant of $M_{P F}$ (including the features mentioned previously regarding multiple occasions, discrete covariates), $M_{I F}$ or $M_{B F}$ for single or multiple-response models. The Simplex algorithm optimizes statistical or exact designs in constrained intervals, given a total number of samples. PFIM uses the Splus function "fun.amoeba" by Daniel Heitjan, which is a translation from the Numerical Recipes [46] for Nelder and Mead's Simplex function [34]. The Fedorov-Wynn algorithm [35, 36, 33] optimizes statistical designs for a given total number of samples among pre-specified sampling times, avoiding clinically unfeasible sampling times. It is written in $\mathrm{C}$ and loaded in PFIM as a dynamic library. Moreover, PFIM uses the function "combn" in the R package combinat to determine all possible elementary designs to be evaluated among the set of allowed sampling times. In PFIM 4.0, optimization can be performed assuming some fixed sampling times. The best one-group population design is the one maximizing the determinant of the FIM of every one-group design defined from all possible elementary protocols. It is obtained by combinatorial optimization and is given by default when running the Fedorov-Wynn algorithm (before calling the dynamic library). This is the approach used for individual design and Bayesian individual design optimization. Of note, there is no limit to the number of sampling points or subjects in PFIM. However, if the design is not enough informative to identify all parameters of the specified model, it could result in a singular information matrix. Moreover, in Fedorov-Wynn algorithm, if the required number of samples for the optimal design and the vector of allowed samples result in too many elementary designs to be evaluated, it may be time consuming especially for complex models and/or written in ordinary differential equations.

New outputs have been added regarding the FIM such as eigenvalues, conditional numbers, the option to save the matrix obtained after evaluation or optimization in a file. Predicted shrinkage is also reported when evaluating or optimizing Bayesian individual design. Evaluation and optimization of the best one-group protocol for population design can be performed accounting for previously obtained results, which can be summarized through a predicted or an observed FIM. Nevertheless, this feature is not yet included in the Fedorov-Wynn algorithm to optimize both the design structure and samplings. It is also possible to visualize the graphs of the model and the 
sensitivity functions without performing evaluation or optimization.

\subsection{Components of the PFIM 4.0 program}

PFIM 4.0 packaged in a zip file can be downloaded from the webpage www.pfim.biostat.fr. It includes two main folders called "Program" and "Examples" as well as three extensive documentations: the user-guide PFIM4.0_UserGuide.pdf, the library of models PFIM_PKPD_Library.pdf and the example document PFIM4.0_Examples.pdf. All the core functions needed to compute the FIM and evaluate or optimize designs are implemented in several R-scripts of the "Program" folder. The files to be filled in by the user are the model.r and the stdin.r files. The model.r file contains equations of the structural model. The stdin.r file contains input information such as the user's knowledge on model parameter values and the specification of the designs to be evaluated or the constraints for the optimization. The path to the folder of the model.r and stdin.r files and to the "Program" folder has to be specified in the PFIM.r file in which the main function PFIM() is implemented. The results obtained after calling this function for the specified inputs are written into stdout.r.

\subsection{Structural model specification}

The structural model to be specified in the model.r file can be expressed either through an analytical form or as a solution of a system of differential equations. PFIM 4.0 provides libraries of pre-implemented models, and users may also define their own model analytically or through a system of differential equations.

\subsubsection{Library of models}

The PFIM library of models implements R expressions or differential equation systems for PKPD models in several R files available in the Program folder. The user has to assign the path of these files in the model.r file in order to use this library. This PKPD library has been available in the PFIM program since PFIM version 3.2 and PFIM Interface version 3.1. Documentation is available and reports the analytical expressions of these models, which have been derived from the PKPD library developed by Bertrand and Mentré [47] for the MONOLIX software (www.lixoft.com/products/monolix).

The PK model library, implemented in the file LibraryPK.r of the Program folder, includes one-, two- and three- compartment models with linear elimination (written using an analytical 
form through an R expression) and with Michaelis-Menten elimination (written using a differential equation system). The list of all the available PK models is given in Tables S1 and S2 of the Supplementary Material, including their characteristics according to: the type of input (bolus, infusion, first-order absorption), the type of elimination (linear or Michaelis-Menten), the number of compartments (one, two or three), the parametrization and the type of administration (single dose, multiple dose or steady state). Presently, there is no model with lag time in this library.

The PD model library supports immediate response models (either as a function of observed concentrations or linked to a pharmacokinetic model) and the turnover response models linked to pharmacokinetic models. Linear, quadratic, logarithmic, Emax, sigmoid Emax, Imax, sigmoid Imax models with null or constant baseline are available to describe an immediate pharmacodynamic response alone and are implemented in closed form in the file LibraryPD_PDdesign.r of the Program folder. For these models, the design variables are the concentrations or the doses instead of the sampling times.

Regarding PD models linked to PK models, there are in PFIM 4.0 four cases to compose the PKPD model depending on the form for each submodel: either with an analytical form (AF) or an ordinary differential equation system (ODE): 1) PK model with linear elimination (AF) and immediate response PD model (AF); 2) PK model with linear elimination (AF) and turnover response PD model (ODE); 3) PK model with Michaelis-Menten elimination (ODE) and immediate response PD model (AF); 4) PK model with Michaelis-Menten elimination and turnover response PD model (ODE). The lists of the available PD models are given in Tables S3, S4 and S5 of the Supplementary Material.

\subsubsection{User-defined models}

In the previous versions of PFIM, a model defined in analytical form by the user needed to be specified through an $\mathrm{R}$ expression. An alternative way to write the model is now available in PFIM 4.0, through an $\mathrm{R}$ function with arguments that are the vector of parameters of the structural model, the times and/or the doses. This feature is used in the first illustrative example of Section 4. Users can also define their model using a system of differential equations in a format suitable for the function "lsoda" of the solver package deSolve.

More details of how to specify models are given in the user guide PFIM4.0_User_guide.pdf 
available when downloading PFIM 4.0.

\subsection{Input file}

The inputs for PFIM 4.0 are entered through specific $\mathrm{R}$ variables by filling in the input file called by default stdin.r. In the first section of this input file, the user has to specify "EVAL" or "OPT" for design evaluation or optimization, respectively, as well as the type of the FIM ( $M_{P F}$, $M_{I F}$ or $M_{B F}$ ) corresponding to the type of design studied (population or individual or Bayesian design, respectively). The user can choose to save the evaluated or optimized FIM and can load previous information through a predicted or observed FIM stored in a file, by specifying its file name as input. The FIM computed by PFIM 4.0 has a block diagonal expression by default (option 1). The number of responses must correspond to those specified in the structural model written in the model.r file.

The second section of the input file is composed of general parameters required for both evaluation and optimization, such as the model form (analytical expression model or differential equation model), according to which, the user has to fill in corresponding specifications. The parametrization of the structural model, the inter-individual or inter-occasion variance model (additive or exponential), the values of the fixed effect parameters (fixed or estimated), of the variance terms including inter-individual variability and inter-occasion variability (when the number of occasions is greater than 1) as well as parameters for the residual error model are required. To take into account discrete covariates (changing or not with occasion), the user has to specify the corresponding parts. The user has to specify the design to evaluate or, for optimization, an initial design. In the case of population design, it is a group of elementary designs, each one associated with a number of subjects. In the case of individual or Bayesian individual design, only an elementary design to be performed in one subject is considered.

The third section of the input file is dedicated to the design optimization, where different algorithm parameters are written. First, the user specifies whether or not the same sampling times are required for each response. Then, the algorithm is chosen: Fedorov-Wynn or Simplex. The Fedorov-Wynn algorithm finds optimal times among vectors of allowed sampling times proposed by the user. In PFIM 4.0, optimization with the Fedorov-Wynn algorithm can be performed assuming that some sampling times are fixed. The Simplex algorithm optimizes the sampling times in given continuous intervals. The user has to specify the minimum delay between two sampling 
times and the time intervals. These specifications apply to optimization of either the population or the Bayesian/individual design. Of note, in order to obtain the best one-group population design and the best individual design, only the combination/evaluation step preceding the Fedorov-Wynn algorithm is used (without calling the dynamic library). The information matrices corresponding to every one-group design from all possible elementary protocols are computed and compared in terms of D-optimality criterion.

Graphical representations are provided if the corresponding option parameters are filled in. Previous PFIM versions already allowed drawing of the graph of the predicted output(s) and the sampling times. PFIM 4.0 now also allows drawing of the graphs of the sensitivity functions (firstorder derivation of the model) with respect to each parameter. These plots can give a good idea about the informative points at which the magnitude of the curves is the greatest. The user can choose to display only graphs of models and/or sensitivity functions before computing the FIM and therefore without performing evaluation or optimization.

Once the model file and the input file are filled in, the user can run the function PFIM 4.0 by calling the function in the R Console window: PFIM ().

\subsection{Output file}

The results are written into an output file named by default Stdout.r or with a name specified in the input file. As in the previous versions, PFIM 4.0 returns a summary of the input, including the the number of responses, the number of occasions in the case of crossover designs, the population or individual/Bayesian design evaluated or the initial design for optimization, the doses or initial conditions (in the case of models defined by the differential equation system), inter-individual variability or inter-occasion variability model and residual error model for each response, covariate model if specified in the input file, error tolerances for the solver of the differential equations system if used, and the name of the previous FIM if considered.

When design optimization is performed, the list of the algorithm options and the optimized protocol are reported in the output file. The file also includes the corresponding population, individual or Bayesian FIM, the name of the file where the FIM is saved, the value of each parameter with the corresponding expected SE and RSE. In the case of Bayesian design, the corresponding shrinkage values are reported. The output file also reports the value of the determinant of the FIM 
corresponding to the evaluated design or the optimized design as well as the D-optimality criterion (i.e. the determinant of the FIM normalized by the number of parameters $P$ to be estimated, $\left.\operatorname{det}(F I M)^{1 / P}\right)$. The eigenvalues of the FIM and the conditional numbers (ratio of maximal over minimal eigenvalues) as well as the correlation matrix are also given by default.

If a graph has been supplied in the input file, the curves of the models and/or the sensitivity functions are plotted. Examples of PFIM 4.0 output file will be shown in Section 4.

\section{Illustration}

We illustrated the use of the new features in PFIM 4.0 by means of two examples. In the first example, we took into account available information from a previous PK study when designing a subsequent study on the same drug. This new feature is particularly useful in adaptive designs. In the second example, we used the Bayesian individual FIM to evaluate and optimize designs for MAP estimation of individual parameters in a dose-response study. This new feature is particularly useful for selecting informative sampling times in therapeutic drug monitoring.

\subsection{Population design for a PK study accounting for previous information}

In this example, we used PFIM 4.0 to determine the best-one group population design taking into account available previous information which was loaded through a FIM.

\subsubsection{Model and methods}

We considered a one-compartment PK model with first-order linear absorption and elimination after single dose administration,

$$
f\left(k_{a}, V, k, t\right)=\frac{d}{V} \frac{k_{a}}{\left(k_{a}-k\right)}\left(e^{-k t}-e^{-k_{a} t}\right)
$$

where $d$ is the dose, $k_{a}$ is the absorption rate constant, $V$ is the apparent volume of distribution and $k$ is the elimination rate constant. The vector of fixed effects $\mu$ is $\left(\mu_{k_{a}}, \mu_{V}, \mu_{k}\right)=(2,15$, 0.25). We assumed an exponential random effect model with a diagonal variance-covariance matrix

$\operatorname{diag}(1,0.1,0.25)$ and a combined residual error model with $\sigma_{\text {inter }}=0.5$ and $\sigma_{\text {slope }}=0.15$. This example involved 50 subjects who received a dose of 100 (dose units). 
A previous PK study on the same drug was conducted in 50 subjects, according to the following design composed of four groups: $\xi=(0.33,1,1.5) \mathrm{h}$ for 13 subjects, $\xi=(1.5,8,12) \mathrm{h}$ for 10 subjects, $\xi=(0.33,8,12)$ h for 9 subjects and $\xi=(0.33,1.5,8) \mathrm{h}$ for 18 subjects. Considering available information from this previous PK study, we wanted to propose the best one-group population design composed of 3 sampling times from the set $(0.33,1,1.5,3,5,8,12) \mathrm{h}$. We used PFIM 4.0 to find the best one-group design accounting for the FIM obtained from the previous study described above. The resulting design was compared with the one optimized without previous information in terms of allocation of optimal times. Both designs were then evaluated for their performance through predicted RSE of population parameters and criterion value.

The text S1 (Supplementary material) is the input file for the evaluation of the best-one group design obtained considering previous information.
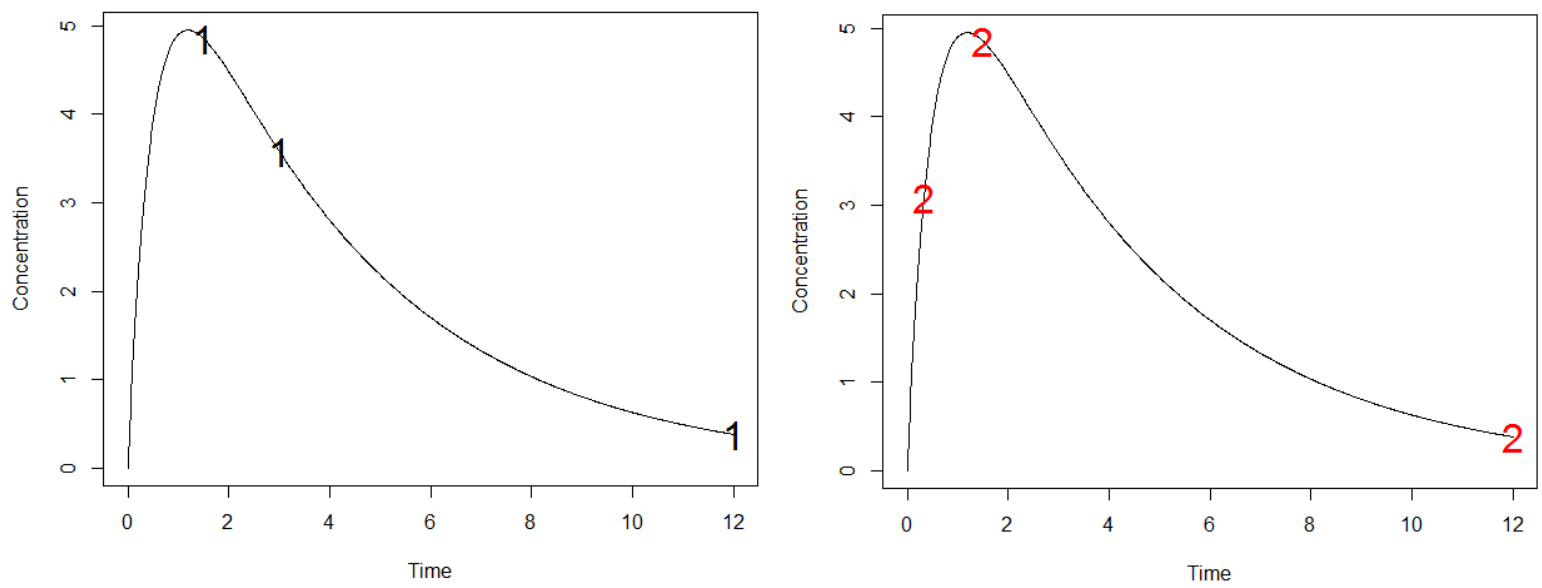

Figure 1: The concentration versus time curve predicted by the one-compartment pharmacokinetic model with first-order linear absorption and elimination. Symbols 1 correspond to the best-one group design $\xi=$ $(1.5,3,12) \mathrm{h}$ obtained by considering previous information (left). Symbols 2 correspond to the design $\xi=$ $(0.33,1.5,12) \mathrm{h}$ obtained without previous information (right).

\subsubsection{Results}

The best one-group design is $\xi=(1.5,3,12) \mathrm{h}$ when considering previous information versus $\xi=(0.33,1.5,12) \mathrm{h}$ without previous information (Figure 1). By evaluating the performance of these two designs, as expected, overall the RSEs are lower and the criterion value is higher when taking into account previous information. Figure 2 displays the output files of the evaluation of the best-one group design obtained for 50 subjects considering previous information versus without 
previous information.

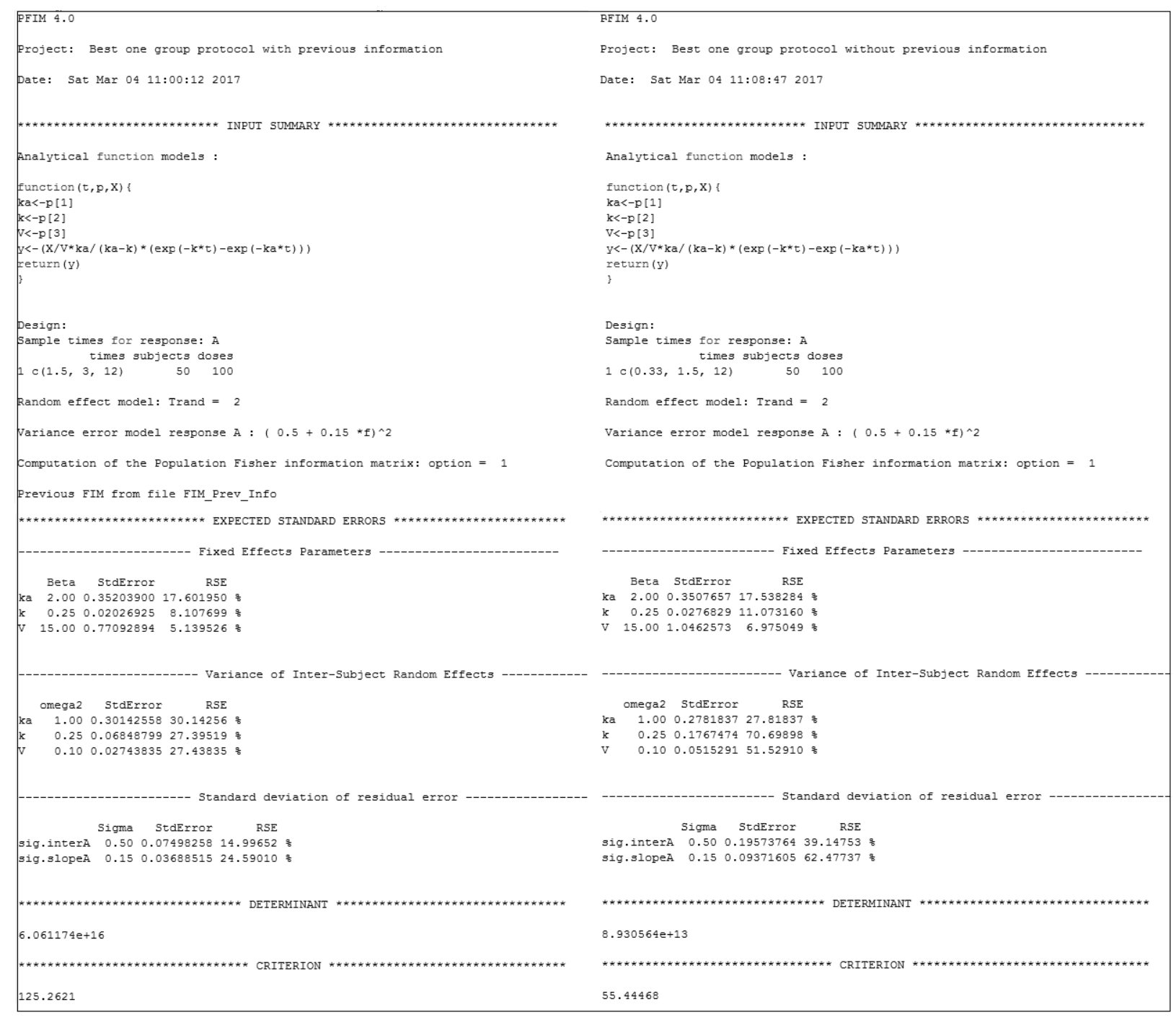

Figure 2: Excerpts of PFIM 4.0 output files for best-one group population design evaluation with previous information loaded though a Fisher information matrix (left) versus without previous information (right).

\subsection{Bayesian individual design for a dose-response study}

In this example, we used PFIM 4.0 to design a new dose-response trial based on a model inspired by [48]. We evaluated the influence of design (number and allocation of doses) and of different variability levels on the precision of estimation and shrinkage of individual parameters.

\subsubsection{Model and methods}

We considered an Emax model (Figure 3) describing the effect of a drug at different doses $d_{j}(j=1, \ldots, n)$ as a function of three parameters: the maximal increase $E_{\text {max }}$ of the effect from 
baseline, the baseline effect $E_{0}$ and the dose $D_{50}$ corresponding to $50 \%$ of the maximal increase of the effect from baseline,

$$
f\left(E_{\max }, D_{50}, E_{0}, d_{j}\right)=E_{0}+\frac{E_{\max } d_{j}}{D_{50}+d_{j}}
$$

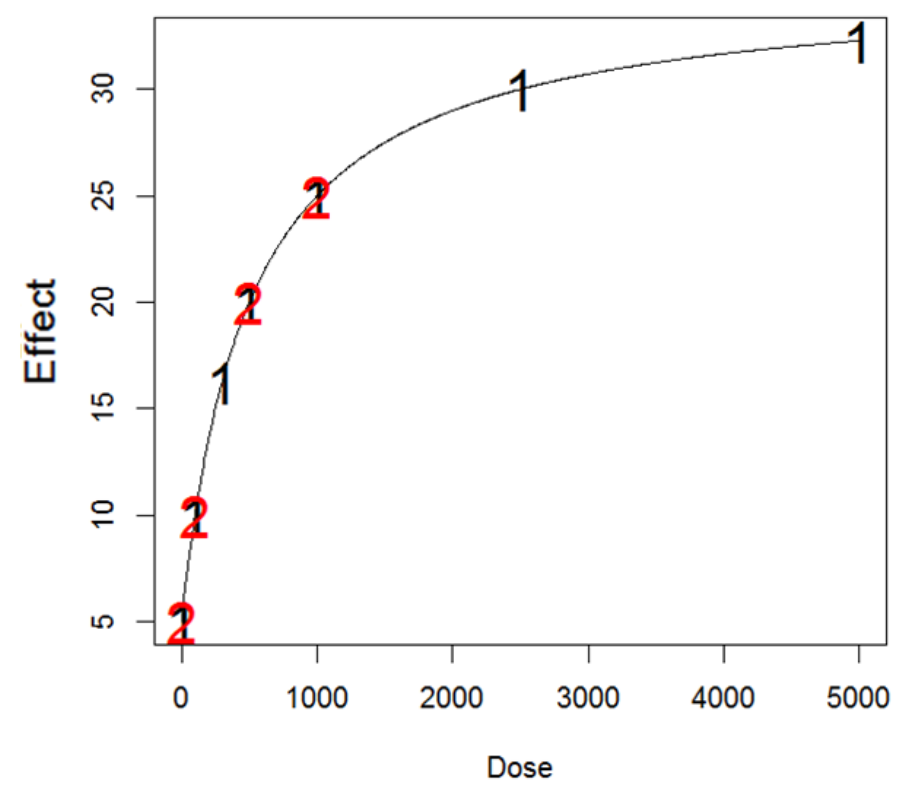

Figure 3: The dose-response curve predicted by the $E_{\max }$ model. Symbols 1 and 2 correspond to the studied rich $(n=7)$ and reduced designs $(n=4)$, respectively.

The vector of fixed effects $\mu$ is $\left(\mu_{E_{\max }}, \mu_{D_{50}}, \mu_{E_{0}}\right)=(30,500,5)$. As in [48], we considered an exponential random effect model with a diagonal variance-covariance matrix of diagonal terms $\omega^{2}$ as well as an additive residual error model with $\sigma_{\text {inter }}=\sigma$ and $\sigma_{\text {slope }}=0$. Two levels of inter-individual variability and residual error variability were studied: $\omega=30 \%$ versus $70 \%$ and $\sigma=1$ versus 2. Two designs were studied: a rich protocol with $n=7$ doses $\xi=$ $(0,100,300,500,1000,2500,5000)$ versus a reduced one with $n=4$ doses $\xi=(0,100,500,1000)$ dose unit.

For each one of the eight scenarios corresponding to different studied values of $(n, \omega, \sigma)$, we used PFIM 4.0 to perform a Bayesian design evaluation: to predict the RSE for individual parameters by $M_{B F}$, to compare them with those obtained by $M_{I F}$ and to compute the expected shrinkage by $M_{B F}$. We also performed Bayesian individual design optimization, with a view to choosing 4 optimal doses among the 7 doses of the rich design, for the standard scenario where $\omega=30 \%$ and 
$\sigma=1$. The Text S2 (Supplementary material) is the input file for this optimization in PFIM 4.0.

\subsubsection{Results}

Figure 4 (Panel A) reports the RSE of parameter $D_{50}$ predicted from $M_{B F}$ vs $M_{I F}$, for different numbers of doses $(n)$ and different levels of variability. Overall, with a sparser design or higher residual error level $(\sigma)$, the RSE predicted either by $M_{B F}$ or $M_{I F}$ increased. However, the influence of losing informativeness, either by reducing the number of samples or increasing the error levels, on the RSE was much lower for $M_{B F}$. With higher inter-individual variability ( $\omega$ ), the RSE obtained with $M_{B F}$ increased but those predicted by $M_{I F}$ remained unchanged as expected. Here, the results showed the advantage of the Bayesian approach in estimating individual parameters especially in the case of sparse design and with high residual variability. Figure 4 (Panel B) shows the shrinkage of parameter $D_{50}$ predicted from $M_{B F}$ for different evaluation scenarios. As expected, the shrinkage increased with the loss of information, i.e., as the design became sparser or residual error level increased. In contrast, as shrinkage quantifies the ratio between individual information and interindividual variability, it decreased when $\omega$ increased. Similar conclusions can be drawn regarding other parameters (not shown).

The best protocol with 4 doses, optimized using PFIM 4.0 for $\omega=30 \%$ and $\sigma=1$, comprised the doses $(0,300,500,5000)$. This design was much more informative than the initial design $(0$, $100,500,1000)$ previously evaluated, with $\operatorname{RSE}\left(D_{50}\right)$ equal to $13.9 \%$ vs $22.8 \%, \operatorname{Sh}\left(D_{50}\right)$ equal to $21.6 \%$ vs $57.8 \%$ and the D-optimality criterion equal to 0.0777 vs 0.0485 , respectively. Therefore, design optimization using $M_{B F}$ in PFIM 4.0 allows us to find an informative sparse design, giving reasonable RSE and shrinkages. An excerpt of the PFIM 4.0 output file showing the results with this optimal design is given in Figure 5.

\section{Discussion}

This paper presents all the available features in PFIM 4.0, the newest version of the $\mathrm{R}$ program PFIM for design evaluation and optimization in NLMEMs. The methodology is based on the Fisher information matrix, evaluated using a linear approximation of the model, first proposed for single response models by [14], and extended to account for multiple response models by [16] (implemented in PFIM since version 3.0) as well as for inter-occasion variability and discrete covariates 


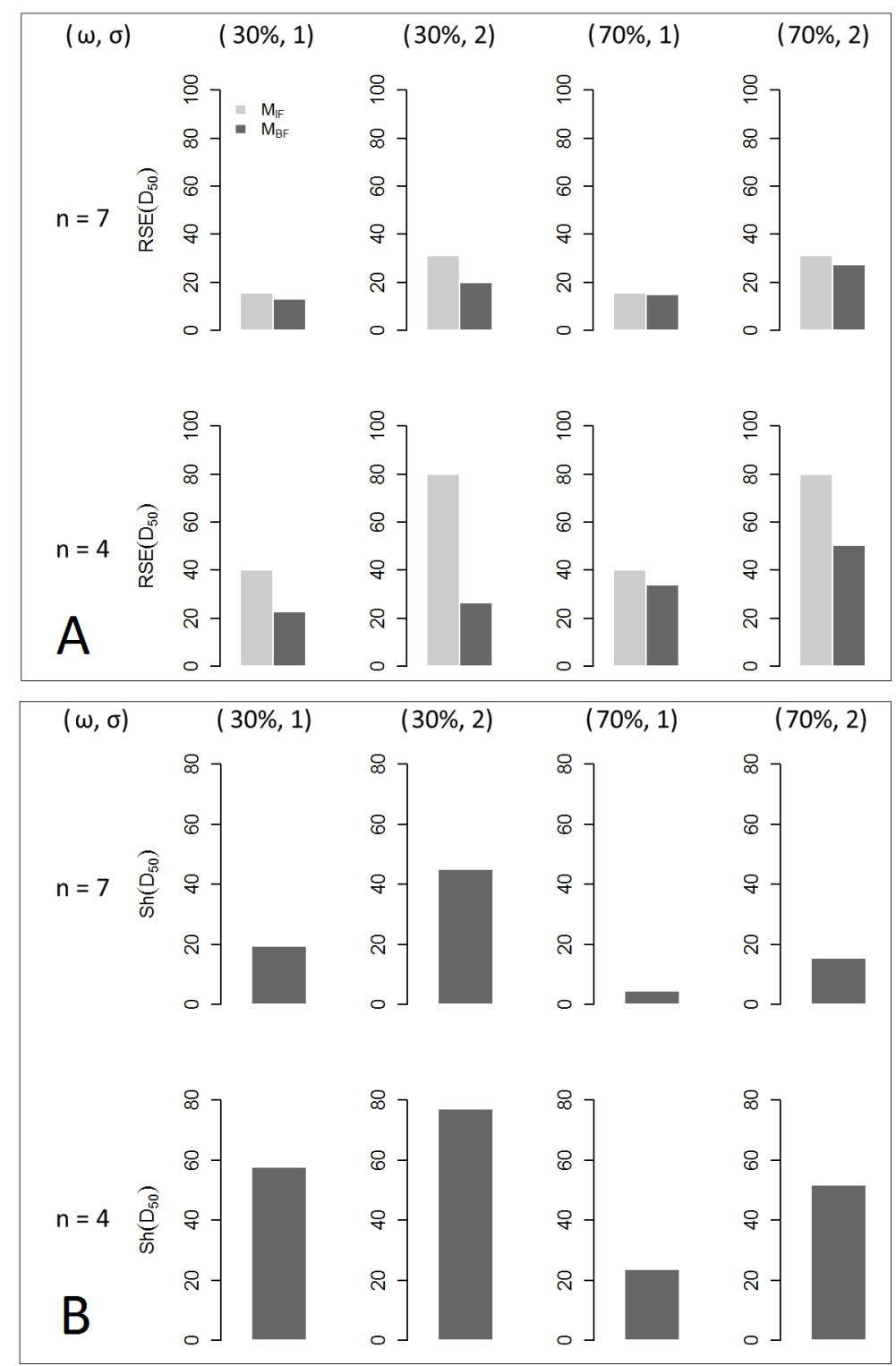

Figure 4: Panel A: Relative standard error (RSE, \%) of parameter $D_{50}$ predicted from the individual Fisher information matrix $M_{I F}$ (light grey bar) versus the individual Bayesian information matrix $M_{B F}$ (dark grey bar); Panel B: Shrinkage (Sh,\%) of parameter $D_{50}$ predicted from $M_{B F}$ (dark grey bar). The results were obtained for different number of doses $(n)$ and different levels of inter-individual $(\omega)$ and residual $(\sigma)$ variability.

by [33] and [19] (since version 3.2). The current version 4.0 includes several new features. First, regarding model specification, since version 3.2, the library of PK models has included one-, twoand three- compartment models with linear elimination and with Michaelis-Menten elimination. A library of PD models is also available. Besides using the library of models and user-defined models written as R expressions or as differential equation systems, a new way has been added to specify models through R functions. Furthermore, PFIM now allows evaluation and optimization assuming 


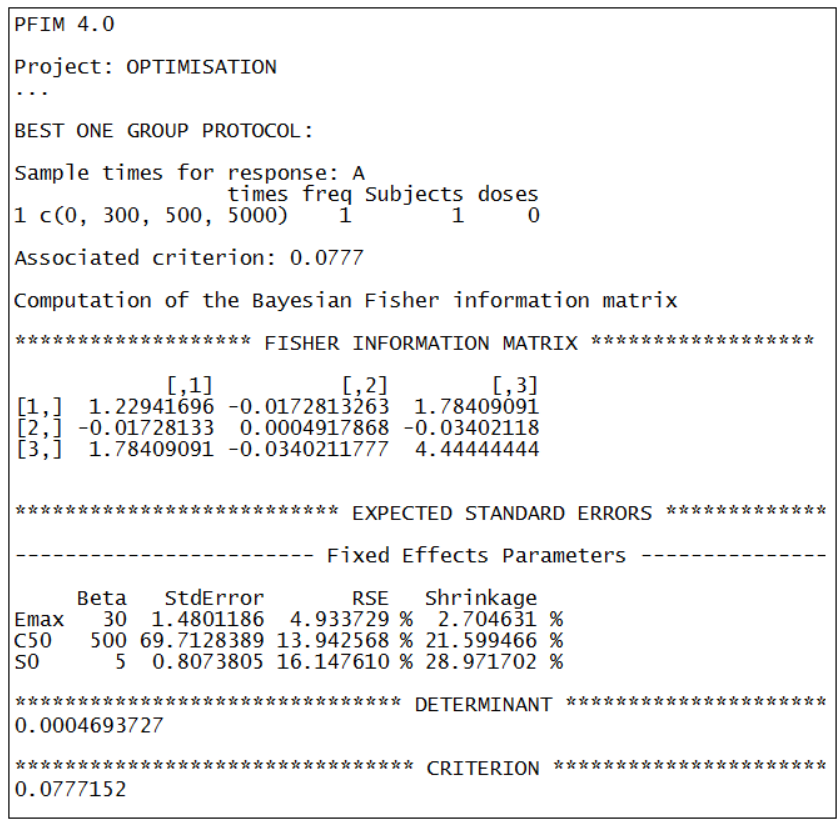

Figure 5: Excerpt of a PFIM 4.0 output file for individual Bayesian design optimisation to choose 4 optimal doses among 7 doses and the associated relative standard error (RSE), shrinkage and D-optimality criterion.

some parameters are fixed. Regarding optimizations, both the Fedorov-Wynn and Simplex algorithms have been available for D-optimal designs since version 3.0. Each algorithm corresponds to a different optimization strategy. The former chooses optimal points among those specified by the user, while the latter optimizes within continuous intervals which can be useful to explore all the design space. However, the Simplex algorithm can be much slower than Fedorov-Wynn algorithm and sometimes result in sampling times that have no clinical relevance. By default, the best-one group protocol when using Fedorov-Wynn algorithm is always given in this new version, which can be useful to simplify logistical aspects in clinical practice. Moreover, PFIM now allows optimization with some times fixed in the Fedorov-Wynn algorithm. New outputs regarding the FIM, such as eigenvalues, conditional numbers, the option to save the matrix obtained after evaluation or optimization in a file, are now available. Previous obtained information can be taken account by loading a predicted or an observed FIM, which is important when performing adaptive designs. The population of a previous study providing this amount of information, which obviously depends on its sample size, can be different from the population of the new study to be designed. In addition, the Bayesian individual FIM has been implemented, taking into account a priori distribution of random effects, in order to find informative designs for MAP estimation of individual parame- 
ters. These designs can now be evaluated or optimized and the predicted shrinkage is also reported, which is useful in therapeutic drug monitoring. It is also possible to visualize the graphs of the model and the sensitivity functions without performing evaluation or optimization.

We illustrated the use of some new features for a PK and a PD study. In the PK example, available information from a previous study saved through the FIM was taken into account in PFIM 4.0 when designing a new study. The best-one group design was different when accounting or not for previously available information. Not surprisingly, since the previous information was obtained from a design with early time points, these times disappeared from the best one group design obtained when considering previous information. By comparing the estimation precision with the two optimized designs, we showed that the precision could be improved with additional information from previous studies, which highlighted the advantage of adaptive designs. In the PD example, PFIM 4.0 was used to evaluate and optimize Bayesian individual designs, showing that the Bayesian FIM enables efficient prediction of estimation precision and shrinkage for individual parameters obtained by MAP $[21,5]$. This new feature in PFIM 4.0 is useful to select informative sampling times in therapeutic drug monitoring.

PFIM 4.0 implements an approximate population and Bayesian individual FIM to evaluate and optimize designs. The relevance of this approximation, based on a linearization of the model, has been shown by clinical trial simulation in several publications based on examples from continuous data $[16,19,18,39]$. However, the linearization has some limitations in the case of complex nonlinear models with large variability and in mixed models for discrete data, especially for sparse designs, as shown in $[49,39]$. Alternatives using Monte Carlo and Adaptive Gaussian Quadrature [38] or using Monte Carlo and Hamiltonian Monte Carlo (available in R package MIXFIM for design evaluation) [39], which work well for both continuous and discrete data models have been proposed; they have not yet been integrated into PFIM. A limitation of the design methods based on the expected FIM currently implemented in all design software programs or clinical trial simulation is that they require knowledge of the model and its parameters, which leads to designs that are only locally optimal. Robust designs (with respect to model and/or parameters) [50,51] and/or adaptive designs $[29,28]$ are the solutions to overcome this limitation.

We plan to include other new developments and extensions in future versions of PFIM: allowing non-diagonal variance-covariance matrix of the random effects for correlation between these 
random effects [52], accounting for fixed effects for the influence of continuous covariates, including the possibility for the user to specify cost functions in the Fedorov-Wynn algorithm to penalize less feasible designs [53], taking into account the probability of data being observed or below the limit of quantification at each sampling time $[4,54]$. It would also be interesting to include in PFIM different optimality criteria and to explore alternative algorithms for finding optimum designs such as multiplicative algorithms [55] or discrete particle swarm optimization [56]. Presently, the PFIM group is working on implementing PFIM into a proper package in object-oriented R (S4) which will facilitate addition of all these new features, including the Hamiltonian Monte Carlo and Adaptive Gaussian quadrature methods for evaluating the FIM.

In parallel to PFIM 4.0, PFIM Interface 4.0, an extension of the graphical user version PFIM Interface 3.1 including several new features based on the R script program of PFIM 4.0, has recently been proposed and is available for downloading on the PFIM website. These works were in part performed through the DDMoRe (Drug Disease Model Resources) project (www.ddmore.eu). One of the main objectives of the project was to develop languages and tools facilitating modelling and simulation activities. The PFIM group contributed to the methodology of optimal design [23], to the definition of the language for design elements and to the requirements for a converter to PFIM allowing workflows to be streamlined [57].

In conclusion, the implementation of the FIM in NLMEMs proposed in PFIM 4.0 is a useful tool for the evaluation and optimization of designs in the expanding development of longitudinal studies in pharmacometrics. PFIM 4.0 is distributed under the terms of the GNU General Public License (GNU GPL), version 3 or later. PFIM functions, including the user-guide, the documentations of all implemented PK/PD models and a large number of illustrative examples, are freely available from the PFIM website: http://www.pfim.biostat.fr.

\section{Acknowledgements}

The works leading to these results were supported in part by the Innovative Medicines Initiative Joint Undertaking under grant agreement number 115156, resources of which are composed of financial contributions from the European Union's Seventh Framework Programme (FP7/2007-2013) and EFPIA companies' in kind contribution. The DDMoRe project is also financially supported by contributions from Academic and Small Medium Enterprise partners. These works have also 
received support from the European Unions 7th Framework Programme for research, technological development and demonstration under grant agreement number 602552. The authors thank François Cohen for his help with the CATIBioMed cluster.

\section{Conflict of interest statement}

The authors declare no conflict of interest.

\section{References}

[1] Mould DR, Upton RN. Basic concepts in population modeling, simulation, and model-based drug development. CPT: Pharmacometrics \& Systems Pharmacology 2012; 1:e6.

[2] Mould DR, Upton RN. Basic concepts in population modeling, simulation, and model-based drug development - part 2: Introduction to pharmacokinetic modeling methods. CPT: Pharmacometrics \& Systems Pharmacology 2013; 2:e38.

[3] Mould DR, Upton RN. Basic concepts in population modeling, simulation, and model-based drug development - part 3: Introduction to pharmacodynamic modeling methods. CPT: Pharmacometrics \& Systems Pharmacology 2014; 3:e88.

[4] Dumont C, Mentré F, Gaynor C, Brendel K, Gesson C, Chenel M. Optimal sampling times for a drug and its metabolite using SIMCYP simulations as prior information. Clinical Pharmacokinetics Jan 2013; 52(1):43-57.

[5] Nguyen TT, Bénech H, Pruvost A, Lenuzza N. A limited sampling strategy based on maximum a posteriori Bayesian estimation for a five-probe phenotyping cocktail. European Journal of Clinical Pharmacology 2016; 72(1):39.

[6] Madelain V, Oestereich L, Graw F, Nguyen THT, de Lamballerie X, Mentré F, Gunther S, Guedj J. Ebola virus dynamics in mice treated with favipiravir. Antiviral Research 2015; 123:70-77. 
[7] Nguyen TT, Guedj J, Chachaty E, de Gunzburg J, Andremont A, Mentré F. Mathematical modeling of bacterial kinetics to predict the impact of antibiotic colonic exposure and treatment duration on the amount of resistant enterobacteria excreted. PLoS Computational Biology 2014; 10(9):e1003 840.

[8] Pinheiro J, Bornkamp B, Glimm E, Bretz F. Model-based dose finding under model uncertainty using general parametric models. Statistics in Medicine 2014; 33:1646-1661.

[9] van der Graaf PH. CPT: Pharmacometrics and Systems Pharmacology. CPT: Pharmacometrics \& Systems Pharmacology Sep 2012; 1(9):e8.

[10] Pillai G, Mentré F, Steimer J. Non-linear mixed effects modelling from methodology and software development to driving implementation in drug development science. Journal of Pharmacokinetics and Pharmacodynamics 2005; 32:161-183.

[11] Atkinson AC, Donev AN, Tobias RD. Optimum Experimental Designs. Oxford Statistical Science, Oxford University Press: Oxford, 2009.

[12] Pronzato L, Pázman A. Design of Experiments in Nonlinear Models. Springer: New York, 2013.

[13] Fedorov VV, Leonov SL. Optimal Design for Nonlinear Response Models. Chapman and Hall/ CRC Press: Boca Raton, 2014.

[14] Mentré F, Mallet A, Baccar D. Optimal design in random effect regression models. Biometrika 1997; 84:429-442.

[15] Hooker A, Vicini P. Simultaneous population optimal design for pharmacokineticpharmacodynamic experiments. American Association of Pharmaceutical Scientists Journal $2005 ; 7: 759-785$.

[16] Bazzoli C, Retout S, Mentré F. Fisher information matrix for nonlinear mixed effects multiple response models: evaluation of the appropriateness of the first order linearization using a pharmacokinetic/pharmacodynamic model. Statistics in Medicine 2009; 28(14):1940-1956. 
[17] Mielke T, Schwabe R. Some considerations on the Fisher information in nonlinear mixed effects models. Proceedings of the 9th International Workshop in Model-Oriented Design and Analysis, Bertinoro, Italy, 2010.

[18] Nyberg J, Bazzoli C, Ogungbenro K, Aliev A, Leonov S, Duffull S, Hooker A, Mentré F. Methods and software tools for design evaluation for population pharmacokineticspharmacodynamics studies. British Journal of Clinical Pharmacology 2014; 79:6-17.

[19] Nguyen TT, Bazzoli C, Mentré F. Design evaluation and optimisation in crossover pharmacokinetic studies analysed by nonlinear mixed effects models. Statistics in Medicine 2012; 31(11-12):1043-1058.

[20] Merlé Y, Mentré F. Bayesian design criteria: computation, comparison, and application to a pharmacokinetic and a pharmacodynamic model. Journal of Pharmacokinetics and Biopharmaceutics 1995; 23(1):101-125.

[21] Combes F, Retout S, Frey N, Mentré F. Prediction of shrinkage of individual parameters using the Bayesian information matrix in non-linear mixed effect models with evaluation in pharmacokinetics. Pharmaceutical Research 2013; 30:2355-2367.

[22] Savić R, Karlsson M. Importance of shrinkage in empirical Bayes estimates for diagnostics: problems and solution. American Association of Pharmaceutical Scientists Journal 2009; 11:558-569.

[23] Mentré F, Chenel M, Comets E, Grevel J, Hooker A, Karlsson MO, Lavielle M, Gueorguieva I. Current use and developments needed for optimal design in pharmacometrics: A study performed among DDMoRe's european federation of pharmaceutical industries and associations members. CPT: Pharmacometrics \& Systems Pharmacology 2013; 2:e46.

[24] Dodds MG, Hooker AC, Vicini P. Robust population pharmacokinetic experiment design. Journal of Pharmacokinetics and Pharmacodynamics ; 32(1):33-64.

[25] Foo LK, McGree J, Eccleston J, Duffull S. Comparison of robust criteria for D-optimal designs. Journal of Biopharmaceutical Statistics 2012; 22(6):1193-1205. 
[26] Chang M. Adaptive Design Theory and Implementation Using SAS and R. Boca Raton: Chapman and Hall/CRC, 2007.

[27] Foo L, Duffull S. Adaptive optimal design for bridging studies with an application to population pharmacokinetic studies. Pharmaceutical Research 2012; 29:1530-1543.

[28] Lestini G, Dumont C, Mentré F. Influence of the size of cohorts in adaptive design for nonlinear mixed effects models: An evaluation by simulation for a pharmacokinetic and pharmacodynamic model for a biomarker in oncology. Pharmaceutical Research 2015; 32(10):3159_ 3169.

[29] Dumont C, Chenel M, Mentré F. Two-stage adaptive designs in nonlinear mixed effects models: application to pharmacokinetics in children. Communications in Statistics - Simulation and Computation 2016; 45:1511-1525.

[30] Retout S, Duffull S, Mentré F. Development and implementation of the population Fisher information matrix for the evaluation of population pharmacokinetic designs. Computer Methods and Programs in Biomedicine 2001; 65:141-151.

[31] Bazzoli C, Retout S, Mentré F. Design evaluation and optimisation in multiple response nonlinear mixed effect models: PFIM 3.0. Computer Methods and Programs in Biomedicine $2010 ; 98: 55-65$.

[32] Bazzoli C, Nguyen T, Comets E, Dubois A, Le Nagard H, Mentré F. New features for population design evaluation and optimisation with R functions: PFIM Interface 3.1 and PFIM 3.2. Abstract 2088. 20th Meeting of the Population Approach Group in Europe, Berlin, Germany, 2011. https : / /www . page-meeting . org/ ?abstract $=2088$.

[33] Retout S, Comets E, Samson A, Mentré F. Design in nonlinear mixed effects models: optimization using the Federov-Wynn algorithm and power of the Wald test for binary covariates. Statistics in Medicine 2007; 26:5162-5179.

[34] Nelder J, Mead R. A Simplex method for function minimization. Computer Journal 1965; 1:308-313. 
[35] Fedorov V. Theory of Optimal Experiments. Academic Press: New York, 1972.

[36] Wynn HP. Results in the theory and construction of D-optimum designs. Journal of the Royal Statistical Society B 1972; 34:133-147.

[37] Magnus J, Neudecker H. Matrix Differential Calculus with Applications in Statistics and Econometrics. Wiley: New York, 1988.

[38] Ueckert S, Mentré F. A new method for evaluation of the Fisher information matrix for discrete mixed effect models using Monte Carlo sampling and adaptive Gaussian quadrature. Computational Statistics \& Data Analysis 2016; 111:203-219.

[39] Riviere MK, Ueckert S, Mentré F. An MCMC method for the evaluation of the Fisher information matrix for non-linear mixed effect models. Biostatistics 2016; 17(4):737-750.

[40] FDA. Guidance for Industry - Statistical Aproaches to establishing bioequivalence 2001.

[41] EMEA. Note for guidance on the investigation of bioavailability and bioequivalence 2001 .

[42] Panhard X, Taburet AM, Piketti C, Mentré F. Impact of modelling intra-subject variability on tests based on non-linear mixed-effects models in cross-over pharmacokinetic trials with application to the interaction of tenofovir on atazanavir in HIV patients. Statistics in Medicine 2007; 26:1268-1284.

[43] Schuirmann DJ. A comparison of the two one-sided tests procedure and the power approach for assessing the equivalence of average bioavailability. Journal of Pharmacokinetics and Biopharmaceutics 1987; 15:657-680.

[44] Pilz J. Bayesian Estimation and Experimental Design in Linear Regression Models. Wiley: New York, 1991.

[45] Chaloner K, Verdinelli I. Bayesian experimental design: A review. Statistical Science 1995; 10:273-304.

[46] Press WH, Teukolsky SA, Vetterling WT, Flannery BP. Numerical Recipes: The Art of Scientific Computing. 3 edn., Cambridge University Press, 2007. 
[47] Bertrand J, Mentré F. Mathematical expressions of the pharmacokinetic and pharmacodynamic models implemented in the MONOLIX. MONOLIX software. 2008.

[48] Plan EL, Maloney A, Mentré F, Karlsson MO, Bertrand J. Performance comparison of various maximum likelihood nonlinear mixed-effects estimation methods for dose-response models. American Association of Pharmaceutical Scientists Journal 2012; 14(3):420-432.

[49] Nguyen TT, Mentré F. Evaluation of the Fisher information matrix in nonlinear mixed effect models using adaptive Gaussian quadrature. Computational Statistics \& Data Analysis 2014; 80:57-69.

[50] Lestini G, Ueckert S, Mentré F. Robust design in model based analysis of longitudinal clinical data. Presented at PODE meeting, June 20, Uppsala, Sweden. 2016.

[51] Loingeville F, Nguyen TT, Riviere MK, Mentré F. Using Hamiltonian Monte Carlo to design longitudinal studies with discrete data. 154th ICB Seminar - Eleventh International Seminar on Statistics and Clinical Practice, Warsaw, Poland. 2017.

[52] Dumont C, Chenel M, Mentré F. Influence of covariance between random effects in design for nonlinear mixed effect models with an illustration in paediatric pharmacokinetics. Journal of Biopharmaceutical Statistics 2013; 24(3):471-492.

[53] Retout S, Comets E, Bazzoli C, Mentré F. Design optimisation in nonlinear mixed effects models using cost functions: application to a joint model of infliximab and methotrexate pharmacokinetics. Communication in Statistics Part A - Theory and Methods 2009; 38:33513368.

[54] Nguyen THT, Nguyen TT, Mentré F. Individual Bayesian information matrix for predicting estimation error and shrinkage of individual parameters accounting for data below the limit of quantification. Pharmaceutical Research 2017; 34(10):2119.

[55] Yu Y. Monotonic convergence of a general algorithm for computing optimal designs. Annals of Statistics 2010; 38(3):1593-1606.

[56] Chen RB, Chang SP, Wang W, Tung HC, Wong WK. Minimax optimal designs via particle swarm optimization methods. Statistics and Computing 2015; 25(5):975-988. 
[57] Smith MK, Moodie SL, Bizzotto R, Blaudez E, Borella E, Carrara L, Chan P, Chenel M, Comets E, Gieschke R, et al.. Model description language (MDL): A standard for modeling and simulation. CPT: pharmacometrics \& systems pharmacology 2017; 6(10):647-650. 